\title{
Utilities associated with subcutaneous injections and intravenous infusions for treatment of patients with bone metastases
}

This article was published in the following Dove Press journal:

Patient Preference and Adherence

28 August 2013

Number of times this article has been viewed

\author{
Louis S Matza' \\ Ze Cong ${ }^{2}$ \\ Karen Chung ${ }^{2}$ \\ Alison Stopeck ${ }^{3}$ \\ Katia Tonkin ${ }^{4}$ \\ Janet Brown ${ }^{5}$ \\ Ada Braun ${ }^{2}$ \\ Kate Van Brunt ${ }^{6}$ \\ Kelly McDaniel' \\ 'Outcomes Research, United \\ BioSource Corporation, Bethesda, \\ MD, USA; ${ }^{2}$ Amgen, Inc, Thousand \\ Oaks, CA, USA; ${ }^{3}$ Department of \\ Medicine, University of Arizona, \\ Tucson, AZ, USA; ${ }^{4}$ Department of \\ Oncology, University of Alberta, \\ Edmonton, Alberta, Canada; ${ }^{5}$ Leeds \\ Institute of Molecular Medicine, \\ St James University Hospital, Leeds, \\ UK; ${ }^{6}$ formerly with Outcomes \\ Research, United BioSource \\ Corporation, Bethesda, MD, USA
}

Correspondence: Louis S Matza United BioSource Corporation, 7I0I Wisconsin Avenue, Suite 600, Bethesda, MD 20814, USA

$\mathrm{Tel}+\mathrm{I} 3016647263$

Fax +I 3016549864

Email louis.matza@unitedbiosource.com
Introduction: Although cost-utility models are often used to estimate the value of treatments for metastatic cancer, limited information is available on the utility of common treatment modalities. Bisphosphonate treatment for bone metastases is frequently administered via intravenous infusion, while a newer treatment is administered as a subcutaneous injection. This study estimated the impact of these treatment modalities on health state preference.

Methods: Participants from the UK general population completed time trade-off interviews to assess the utility of health state vignettes. Respondents first rated a health state representing cancer with bone metastases. Subsequent health states added descriptions of treatment modalities (ie, injection or infusion) to this basic health state. The two treatment modalities were presented with and without chemotherapy, and infusion characteristics were varied by duration ( 30 minutes or 2 hours) and renal monitoring.

Results: A total of 121 participants completed the interviews (52.1\% female, $76.9 \%$ white). Cancer with bone metastases had a mean utility of 0.40 on a standard utility scale ( $1=$ full health; $0=$ dead). The injection, 30-minute infusion, and 2-hour infusion had mean disutilities of $-0.004,-0.02$, and -0.04 , respectively. The mean disutility of the 30 -minute infusion was greater with renal monitoring than without. Chemotherapy was associated with substantial disutility $(-0.17)$. When added to health states with chemotherapy, the mean disutilities of injection, 30-minute infusion, and 2-hour infusion were $-0.02,-0.03$, and -0.04 , respectively. The disutility associated with injection was significantly lower than the disutility of the 30-minute and 2-hour infusions $(P<0.05)$, regardless of chemotherapy status.

Conclusion: Respondents perceived an inconvenience with each type of treatment modality, but injections were preferred over infusions. The resulting utilities may be used in cost-utility models examining the value of treatments for the prevention of skeletal-related events in patients with bone metastases.

Keywords: skeletal-related event, infusion, injection

\section{Introduction}

When solid tumors metastasize to bone, localized bone destruction occurs, which may result in pain, decreased survival, and complications referred to as skeletal-related events (SREs). ${ }^{1,2}$ These SREs, such as pathological fracture, radiation to the bone, surgery to the bone, and spinal cord compression, can be severely debilitating, with the potential to cause increased pain and functional impairment. ${ }^{3,4}$ Intravenous (IV) bisphosphonates, such as zoledronic acid, have commonly been used for the treatment of patients with bone metastases. The bisphosphonates are bone antiresorptive drugs that reduce osteoclast activity, thus helping to prevent or delay the onset of SREs. ${ }^{5-7}$ Zoledronic acid is administered via IV infusion every 3 to 4 weeks and requires renal 
monitoring, with dose adjustment if renal impairment is present. ${ }^{8}$ Denosumab, which has a targeted mechanism of action distinct from that of bisphosphonates, is a more recently developed bone-modifying agent that is also used to prevent or delay SREs. ${ }^{9,10}$ This newer treatment is a fully human monoclonal antibody against RANK Ligand (RANKL), a key mediator of cancer-induced bone destruction. Denosumab is administered via subcutaneous (SC) injection every 4 weeks, and it does not require renal monitoring. ${ }^{11}$ In clinical trials among patients with solid tumors and bone metastases, denosumab has been found to have superior efficacy for the prevention of SREs compared with zoledronic acid. ${ }^{10,12,13}$

As new treatments such as denosumab are developed, it is important to evaluate their cost-effectiveness relative to existing treatments in order to demonstrate their value to patients, clinicians, reimbursement authorities, and payers. A cost-utility analysis is a type of cost-effectiveness analysis that incorporates the preferences of individuals for different treatment-related outcomes. These preferences are quantified in terms of utilities, which are values representing health status and health-related quality of life (HRQL) on a scale with anchors of 0 representing death and 1 representing full health. Utilities are most often used to represent preferences for health states involving medical conditions and treatment efficacy. However, there is also a substantial body of research in which utilities were used to quantify preferences for treatment attributes that could have an impact on convenience and quality of life. For example, previous studies have found that utilities differ depending on a range of treatment modalities, including surgical vs nonsurgical management; ${ }^{14}$ inhaled vs injected insulin; ${ }^{15}$ oral vs injectable treatment; ${ }^{16-18}$ dose frequency; ${ }^{19,20}$ inpatient vs outpatient treatment; ${ }^{21}$ two types of prenatal genetic testing; ${ }^{22}$ daily aspirin use; ${ }^{23}$ early-stage cervical cancer treatment options; ${ }^{24}$ and specific medication options. ${ }^{25}$ Across these studies, more convenient treatments were consistently associated with greater utility values.

Previous studies have found differences in patient preference associated with SC injections and IV infusions for the treatment of a range of medical conditions. ${ }^{26-28}$ Therefore, it may be important to quantify the potential utility differences between injections and infusions in the context of treatment for patients with bone metastases in order to accurately represent these treatments in cost-effectiveness modeling. The purpose of the current study was to identify the impact of injection or infusion procedures on health state utility in the context of cancer with bone metastases. This study used time trade-off (TTO) interviews with general population respondents in the UK to focus on the utility or disutility associated with each treatment modality, regardless of treatment efficacy, adverse events, or other treatment outcomes.

\section{Methods Development of health states}

The health state vignettes representing cancer with bone metastases and the addition of treatment modalities were drafted based on a literature review and interviews with clinicians. First, a literature review was conducted to identify articles on bone metastases and treatment of SREs to ensure that the health states would be grounded in clinical research. ${ }^{1-7}$ The information obtained from this literature search was used to draft structured interview guides for the clinician interviews.

Telephone interviews were conducted with four clinicians with MD degrees who specialized in oncology. One of these clinicians worked full time in clinical research, focusing on the treatment of patients with bone metastases. The other three clinicians were oncologists who primarily worked in clinical settings, treating patients with bone metastases while participating in clinical research. Each of the four clinicians participated in two telephone interviews before the health states were drafted. During these interviews, they responded to questions regarding administration procedures for denosumab via SC injection and bisphosphonates via IV infusion, either alone or concomitant with chemotherapy. Each clinician described their observations of patients' experiences receiving these treatments as well as the language they use with patients to describe the administration procedures. Although denosumab was not publicly available at the time the study was conducted, all four clinicians had experience with this new treatment. The clinician who worked full time in clinical research had participated in several studies of denosumab, while the other three clinicians had direct experience treating patients with denosumab during clinical trials. The resulting descriptions were incorporated into draft health states.

Then, the four clinicians were interviewed again and asked to comment on how accurately and clearly the draft health states represented patients' treatment experiences. In addition, the clinician who was based in the UK was asked to ensure that all language in the health states was appropriate for UK respondents. The health states were edited until all clinicians agreed that they adequately represented the administration procedures for the relevant treatments.

After the health states were drafted, they were tested in a pilot study conducted with 20 members of the general London population ( 13 women; mean age $=32.4$ years; age 
range $=21$ to 50 ). The draft health states were administered in a TTO interview to ensure that respondents were able to understand the health states and the interview task. The participants generally reported that the health states were clear and easy to understand. Some participants suggested minor revisions in formatting, phrasing, and explanation of the TTO task, and the health states were edited accordingly.

\section{Final health states administered in the time trade-off interview}

The final set of health state vignettes included a "basic health state (health state A)," which was designed to represent a patient with cancer and bone metastases but without description of a treatment. This health state included the following statements:

You have cancer that has spread to your bone. In parts of your body where the cancer has spread, the cancer can weaken your bones. You have pain where the cancer has spread to the bone. This pain is aching and present most of the time. The pain increases with movement, and it may interfere with your daily activities.

These statements had been used in a previous study designed to assess the health state utilities associated with bone metastases and SREs. ${ }^{29}$ In the TTO task, respondents considered living in this health state for a period of 2 years.

An additional nine health states (health states $\mathrm{B}$ to $\mathrm{J}$ ) began with this basic health state, followed by additional statements describing a treatment involving either injection or infusion, in some cases combined with either renal monitoring or chemotherapy. The purpose of this study was to assess preferences for the various treatment modalities, independently of possible differences in treatment efficacy. Therefore, participants were explicitly told that there were no differences in the medical condition among the hypothetical patients described in the health state vignettes. Interviewers provided the following instructions to the participants:

Please do not concern yourself with the effectiveness of the treatment. In all of these health states, you live for two years, and your cancer symptoms are the same. So just try to think about how you would feel about living in each health state for two years, while receiving the treatment as described. The key question is whether the experience of receiving these treatments changes the way you feel about living in health state A. In all health states, your condition remains unchanged for the two-year period.
Health state B included four statements designed to represent injection procedures for denosumab. ${ }^{9,10,12,30}$

You are receiving treatment to stop your bones from becoming weaker and to keep your bone pain from becoming worse. This treatment is a single injection, in which a needle is inserted just under the skin (ie, subcutaneous tissue), in the upper arm, lasting for less than 10 seconds. You may receive this treatment in a hospital outpatient clinic or at your doctor's practice. You receive this treatment once every four weeks.

Health state $\mathrm{C}$ included four statements describing administration procedures for zoledronic acid, a commonly used bisphosphonate. ${ }^{31-33}$

You are receiving treatment to stop your bones from becoming weaker and to keep your bone pain from becoming worse. This treatment is given by intravenous (IV) infusion, which means it is administered directly into the bloodstream through a tiny plastic tube inserted directly into a vein. It takes at least 30 minutes for the administration process. You receive this treatment at a hospital outpatient clinic in an IV treatment room where other patients may be receiving chemotherapy. You receive this treatment once every four weeks.

In health state $\mathrm{C}$, the infusion process was indicated to last at least 30 minutes in order to represent the relatively short infusion time required for zoledronic acid. Infusions of zoledronic acid are recommended to occur over no less than 15 minutes. ${ }^{8,32}$ However, actual times for the entire infusion process have been reported to last somewhat longer, ranging from 13 to 54 minutes. ${ }^{34,35}$ The four clinicians interviewed for the current study diverged on their description of the typical infusion duration, but all agreed that 30 minutes was a reasonable rough estimate. Health state $\mathrm{D}$ was identical to health state $\mathrm{C}$, except for the duration of the infusion process. In health state $\mathrm{D}$, the infusion process was described as lasting 2 hours in order to represent a typical infusion time for pamidronate, another commonly used bisphosphonate, requiring infusion over a longer period of time..$^{34,36}$

During the telephone interviews, the four clinicians agreed with published literature suggesting that blood draws were required for renal monitoring prior to the administration of bisphosphonates. ${ }^{8,37}$ However, opinions varied on whether blood draws occurred more commonly on the same day as the bisphosphonate infusion or on days prior to the bisphosphonate infusion. Therefore, two health states, E and F, were designed to represent these two renal monitoring approaches. 
Health state E was the same as health state C (ie, zoledronic acid), with the addition of statements describing a blood draw on the same day as bisphosphonate treatment:

As part of your treatment plan, you have blood drawn so that your kidney function can be checked to make sure the treatment can be given (each time, the results of the blood draw indicate that you can receive the treatment). This blood draw is done before each IV treatment, and you have to wait two hours for results of the blood draw before receiving the IV treatment.

Similarly, health state F was the same as health state C, except for the addition of statements describing a blood draw 2 days prior to bisphosphonate treatment:

As part of your treatment plan, you have blood drawn so that your kidney function can be checked to make sure the treatment can be given (each time, the results of the blood draw indicate that you can receive the treatment). This blood draw is done two days before receiving each IV treatment, as a separate appointment at the hospital outpatient clinic.

Health state $\mathrm{G}$ described chemotherapy. There are a wide range of chemotherapy administration procedures used in patients with solid tumors that tend to metastasize to bone. ${ }^{38-40}$ Therefore, the four clinicians were questioned multiple times about the details of chemotherapy administration, including duration, frequency, location of IV infusion, and whether chemotherapy and bisphosphonate treatment could be coordinated to be administered during the same appointment. The statements in the chemotherapy health states are based on a consensus from the four clinicians regarding a typical administration approach:

You require chemotherapy to stop the cancer from getting worse, which may have side effects such as hair loss, nausea, and fatigue. The chemotherapy treatment is administered into your bloodstream through an intravenous (IV) infusion, which lasts approximately 1.5 hours. You receive the chemotherapy at a hospital outpatient clinic in an IV treatment room where other patients may be receiving chemotherapy.

You receive the chemotherapy once every four weeks.

The final three health states $(\mathrm{H}, \mathrm{I}$, and $\mathrm{J})$ were designed to identify differences in preference among the treatments associated with bone metastases when they were combined with chemotherapy. Each of these three health states included the chemotherapy statements from health state G. In addition, health state $\mathrm{H}$ included the injectable treatment described in health state B, while health states I and J included the infusions described in health states $\mathrm{C}$ and $\mathrm{D}$, respectively. Health states H, I, and J specify that the treatment associated with bone metastases was administered after the chemotherapy.

The health state used as an upper anchor described a person in full health with no cancer or any other health problems. A “death" health state was used as a lower anchor.

\section{Participants}

All participants were required to be (1) at least 18 years old; (2) able to understand the assessment procedures; (3) able and willing to give written informed consent; and (4) residing in the United Kingdom. Patients were not eligible if they had cognitive impairment, hearing difficulty, visual impairment, severe psychopathology, or insufficient knowledge of English that could interfere with the ability to complete study measures. The TTO interviews were intended to yield utilities that may be used in submissions to agencies like National Institute for Health and Care Excellence (NICE), most of whom prefer that utilities represent general population values. ${ }^{41-43}$ Therefore, the participant inclusion criteria did not specify any particular clinical characteristics.

Participants were recruited through newspaper and online advertisements in Edinburgh and London in July and August of 2011. A total of 462 potential participants responded to the newspaper advertisements by leaving a telephone message, and 225 of these were reached for screening to assess whether they met study inclusion criteria. All 225 potential participants were eligible, and 134 were able to be scheduled for interviews on the days the study was being conducted. A total of 126 participants attended interviews, and 121 of these participants were able to complete the TTO interview.

\section{Utility interview procedures and scoring}

The health states were presented during an interview following a standardized interview script. There were two parts to this interview: First, participants rated the health states using a visual analog scale (VAS) that was intended to introduce participants to the content of the health states. Health states were presented on individual cards to each participant, and the ratings were relative to the anchor states of zero (death) and 100 (full health).

After participants completed the introductory VAS rating task, health state utilities were obtained using the TTO method, which has previously been described in detail. ${ }^{44}$ TTO assessments of health state utilities are often conducted using a 10-year time horizon, as this time horizon was used in the Measurement and Valuation of Health study to elicit 
valuations from the general public for EQ-5D ${ }^{\mathrm{TM}}$ health states. ${ }^{45}$ However, other time horizons may be used, depending on what is most appropriate for the medical condition under examination. Similar to a previous study assessing health state utilities associated with bone metastases, ${ }^{29}$ the TTO task in the current study used a 2-year time horizon so that the impact of the bone treatment modalities would be judged within the context of a realistic life expectancy for a patient with advanced cancer and bone metastases. ${ }^{46}$

In the TTO task, participants were first presented with the basic health state (health state A) and offered a choice between spending 2 years in this health state versus spending varying shorter amounts of time in the full health state, followed by death. After rating health state A, participants rated each of the other health states (health states B-J), in random order, following the same procedures. Participants concluded the TTO task by rating their own current health state.

For each health state rated as preferable to being dead in the TTO task, the utility value was calculated based on the choice in which the respondent is indifferent between $y$ months in the health state being evaluated and $\mathrm{x}$ months in full health (followed by $\mathrm{y}-\mathrm{x}$ months dead). ${ }^{47}$ The resulting utility estimate $(\mathrm{u})$ is calculated as

$$
\mathrm{u}=\mathrm{x} / \mathrm{y}
$$

In the current study, y is 2 years. If participants indicated that a health state was worse than death, the interviewer altered the task so that respondents were offered a choice between immediate death (alternative 1) and a 2-year life span (alternative 2) beginning with varying amounts of time in the health state being rated, followed by full health for the remainder of the 2-year time horizon. For the TTO ratings of health states considered worse than being dead, the current study used a bounded scoring approach, which is commonly used to avoid highly skewed distributions. ${ }^{44}$ This scoring approach limits the score range of health states worse than death to between 0 and -1 . To compute these bounded negative utility values, the current study used the Dolan ${ }^{45}$ method as described by Rowen and Brazier. ${ }^{47}$ This method uses the formula

$$
\mathrm{u}=-\mathrm{x} / \mathrm{t},
$$

where $\mathrm{x}$ is the number of months in full health, and $\mathrm{t}$ is the total life span of alternative 2 in the TTO choice. In the current study, t was 24 months, which is the number of months in the health state being rated plus subsequent months in full health.
The primary purpose of this study was to identify the disutility associated with each type of treatment modality. These disutilities were calculated as the difference between the utility of the basic health state (health state A: metastatic cancer without a treatment modality) and the utility of the otherwise identical health states with an added treatment modality. The calculation of the differences between health states to identify the disutilities of specific attributes has been shown to be useful in other disease areas, such as diabetes. ${ }^{19,48}$

\section{Data collection and statistical analysis procedures}

The one-on-one interviews were conducted in-person in private conference rooms in Edinburgh and London during August 2011. All procedures and instruments were approved by an independent Institutional Review Board (Ethical and Independent Review Services, Corte Madera, CA, USA), and all participants provided written informed consent prior to completing any study measures. Statistical analyses were completed using SAS version 8.12 (SAS Institute Inc., Cary, NC, USA).

Continuous variables including utilities are summarized in terms of means and standard deviations, and categorical variables such as gender and race are summarized as frequencies and percentages. The disutility (ie, utility shift) associated with each treatment modality was calculated by subtracting the utility of each treatment health state $(B-J)$ from the utility of health state A. This disutility quantifies the impact of each treatment modality on preferences for health states in the context of cancer with bone metastases. A series of independent $t$-tests (for continuous variables) and chi-square analyses (for categorical variables) were conducted to compare TTO utilities, utility shifts, and demographic characteristics of the samples from London $(n=57)$ and Edinburgh $(n=64)$, the two cities where data were collected.

Pairwise comparisons between health states, using $t$-tests, were conducted to examine differences in preference for health states differing in treatment modality. The basic health state (health state A) was compared to each health state that included description of a treatment modality (health states B-J). Additional pairwise comparisons were performed to assess differences between the various types of treatments: injection (health state B) vs infusion (C, D); 30-minute infusion (C) vs 2-hour infusion (D); 30-minute infusion (C) vs 30-minute infusion with renal monitoring $(\mathrm{E}, \mathrm{F})$; and injection $(\mathrm{H})$ versus infusion $(\mathrm{I}, \mathrm{J})$ in the context of chemotherapy. 


\section{Results}

\section{Sample description}

The analysis sample includes 121 participants who were able to complete the utility interview (Table 1). The sample had a mean age of 40.8 years $(\mathrm{SD}=13.8)$ and was $52.1 \%$ female. Most of the participants reported their ethnicity as white $(76.9 \%)$, and the majority were employed either full time or part time ( $38.8 \%$ full time and $31.4 \%$ part time). More participants reported being single $(n=69[57.0 \%])$ than mar$\operatorname{ried}(\mathrm{n}=36[29.8 \%])$. Approximately half of the sample had completed a college or university degree $(n=60$ [49.6\%]). When asked to report health conditions, $44.6 \%$ of the sample reported none $(n=54)$. The most commonly reported health conditions were depression $(n=29[24.0 \%])$, hypertension $(n=9[7.4 \%])$, and arthritis $(n=8[6.6 \%])$. Only three participants $(2.5 \%)$ reported that they had a diagnosis of cancer at any time in their lives, and no participants reported cancer that had metastasized to the bone.

There were no significant differences between the London $(n=57)$ and Edinburgh $(n=64)$ samples in age, gender, marital status, employment status, or education level. The only statistically significant difference between the two geographic

Table I Demographic characteristics

\begin{tabular}{ll}
\hline Characteristics & $\begin{array}{l}\text { Descriptive } \\
\text { statistics (n = I2I) }\end{array}$ \\
\hline Age (mean, SD) & $40.8(13.8)$ \\
Gender (n, \%) & \\
Male & $58(47.9 \%)$ \\
Female & $63(52.1 \%)$ \\
Ethnicity (n, \%) & \\
White & $93(76.9 \%)$ \\
Black & $9(7.4 \%)$ \\
Asian & $14(11.6 \%)$ \\
Other & $5(4.1 \%)$ \\
Marital status (n, \%) & \\
Single & $69(57.0 \%)$ \\
Married & $36(29.8 \%)$ \\
Living with partner & $4(3.3 \%)$ \\
Divorced/separated & $12(9.9 \%)$ \\
Employment status (n, \%) & \\
Full-time work & $47(38.8 \%)$ \\
Part-time work & $38(31.4 \%)$ \\
Unemployed & $11(9.1 \%)$ \\
Other* & $25(20.7 \%)$ \\
Education level (n, \%) & \\
Completed college or university degree & $60(49.6 \%)$ \\
Did not complete college or university degree & $61(50.4 \%)$ \\
Location & \\
Edinburgh & $64(52.9 \%)$ \\
London & $57(47.1 \%)$ \\
\hline Note: & \\
\hline
\end{tabular}

Note: *'Other" includes student, homemaker, retired, and disabled. Abbreviation: SD, standard deviation. subsamples was in ethnicity $(P<0.001)$. The Edinburgh sample had a higher percentage of white participants than the London sample (92.2\% vs 76.9\%). In London, eight (14.0\%) participants were black, 11 (19.3\%) were Asian, and four (7.0\%) reported their ethnicity as “other." In Edinburgh, one (1.6\%) participant was black, three (4.7\%) were Asian, and one (1.6\%) reported ethnicity as "other."

\section{Descriptive statistics: VAS ratings and health state utilities}

The VAS scores and TTO utilities for all health states are presented in Table 2. In the total sample, the basic health state (A) describing cancer with bone metastases without treatment had a mean VAS score of 38.8 and a mean TTO utility of 0.40 . Among the nine health states that included a treatment modality (health states B-J), the mean VAS scores ranged from 9.1 to 38.2 , and mean TTO utilities ranged from 0.19 to 0.39 . The respondents' mean ratings of their own current health were 85.5 on the VAS and 0.92 in the TTO task, which reflects a general population sample in relatively good health.

The disutility associated with each treatment modality was computed by subtracting the utility of each health state describing a treatment from the utility of health state A, which is an otherwise identical health state without the inconveniences of a treatment modality (Table 2). Among the health states that did not include chemotherapy, the mean disutilities ranged from -0.004 for a monthly injection to -0.066 for monthly, 30 -minute infusion plus renal monitoring with a blood draw required 2 days prior to the infusion. The health states involving chemotherapy were associated with substantially greater mean disutilities, ranging from -0.175 for chemotherapy alone to -0.211 for chemotherapy in combination with an additional 2-hour infusion.

A series of $t$-tests was conducted to compare utilities and disutilities between the London $(\mathrm{n}=57)$ and Edinburgh $(n=64)$ subsamples. For all health states, the Edinburgh sample had higher utility values than the London sample. The differences between the utilities of the two samples ranged from 0.08 to 0.17 across the ten health states. These differences were statistically significant for three health states (C, D, and F) $(P<0.05)$. However, the pattern of utilities indicating relative preferences among the health states was identical for the two geographic subsamples. Furthermore, there were no significant differences in the disutilities between the London and Edinburgh samples ( $P=0.13$ to 0.58$)$. The differences between the two samples in the disutility associated with each treatment modality were minimal, with differences ranging from 0.01 (health states B and E) to 0.07 (I and J). 
Table 2 Health state VAS ratings and time trade-off utilities

\begin{tabular}{|c|c|c|c|}
\hline \multirow[t]{2}{*}{ Health states } & \multirow{2}{*}{$\begin{array}{l}\text { VAS ratings } \\
(n=I 2 I) \\
\text { mean (SD) }\end{array}$} & \multicolumn{2}{|c|}{ Time trade-off utilities } \\
\hline & & $\begin{array}{l}\text { Health state utility } \\
(n=I 2 I) \\
\text { mean (SD) }\end{array}$ & $\begin{array}{l}\text { Disutility of } \\
\text { each treatment } \\
\text { modality* }\end{array}$ \\
\hline A: Basic health state (cancer with bone metastases, no treatment) & $38.8(19.1)$ & $0.40(0.43)$ & - \\
\hline B: Injection & $38.2(17.4)$ & $0.39(0.43)$ & $-0.004(0.036)$ \\
\hline C: 30 -minute infusion & $33.1(17.0)$ & $0.38(0.44)$ & $-0.023(0.090)$ \\
\hline D: 2-hour infusion & $28.0(17.2)$ & $0.36(0.44)$ & $-0.037(0.106)$ \\
\hline E: 30-minute infusion + renal monitoring (blood draw on same day as infusion) & $25.7(18.0)$ & $0.35(0.43)$ & $-0.050(0.124)$ \\
\hline F: 30 -minute infusion + renal monitoring (blood draw 2 days prior to infusion) & $22.1(16.9)$ & $0.33(0.44)$ & $-0.066(0.122)$ \\
\hline G: Chemotherapy & I7.I (I7.4) & $0.22(0.46)$ & $-0.175(0.225)$ \\
\hline $\mathrm{H}$ : Injection + chemotherapy & I4.I (I7.4) & $0.21(0.46)$ & $-0.190(0.231)$ \\
\hline I: 30 -minute infusion + chemotherapy & $11.6(17.6)$ & $0.20(0.46)$ & $-0.202(0.239)$ \\
\hline J: 2-hour infusion + chemotherapy & $9.1(17.9)$ & $0.19(0.46)$ & $-0.211(0.248)$ \\
\hline Own current health state & $85.5(14.7)$ & $0.92(0.12)$ & - \\
\hline
\end{tabular}

Notes: *This difference score represents the impact of adding each treatment modality to an otherwise identical health state. These values can be interpreted as the "disutility" or utility shift associated with each treatment modality. For health states B to J, the disutility is computed by subtracting the utility of health state A from the utility of each other health state. A negative utility shift indicates that the health state was rated lower than health state $A$.

Abbreviations: SD, standard deviation; VAS, visual analog scale.

\section{Comparisons among health state utilities}

Paired $t$-tests were conducted to examine whether the differences among the health state utilities were statistically significant. The difference between health states $\mathrm{A}$ and $\mathrm{B}$ was not statistically significant $(P=0.25)$. The utilities of all the other health states with treatment modalities $(\mathrm{C}-\mathrm{J})$ were significantly different from the utility of the basic health state without a treatment modality $(t=2.8$ to 9.3$)(P=0.006$ [A vs C]; $P<0.001$ [all other comparisons]).

Paired $t$-tests were also conducted to examine whether the utilities of health states differing by a single treatment attribute were significantly different from each other (Table 3). All differences were statistically significant. For example, the health state describing an injection (health state B) was associated with a significantly higher utility than the health states representing the 30-minute infusion $(P=0.01)$ and the 2 -hour infusion $(P<0.001)$. Adding renal monitoring to the 30-minute infusion resulted in a significantly lower utility $(P<0.001)$ than the 30 -minute infusion alone, regardless of whether renal monitoring occurred on the same day as the infusion (E) or 2 days prior to the infusion (F). There were also significant differences between the 30-minute infusion $(C)$ and the 2-hour infusion (D) $(P=0.002)$, as well as between the two types of renal monitoring (E vs F) $(P=0.005)$.

The differences among the treatment modalities were also statistically significant when presented in combination with chemotherapy (Table 3). The health state representing an injection plus chemotherapy $(\mathrm{H})$ had a significantly lower utility than the health state representing chemotherapy alone (G) $(P=0.004)$. However, the injection health state with chemotherapy had a significantly higher utility than either the 30-minute or 2-hour infusion health states (I and J) (both $P<0.01$ ). These two health states representing infusion plus chemotherapy, which differed only by the duration of infusion, were significantly different from each other $(P=0.003)$.

\section{Discussion}

The results of this study indicate that treatment modality had an impact on preference and utility, independently of treatment effectiveness or tolerability. Although this is the first study assessing utility associated with the characteristics of treatment for patients with bone metastases, the findings are consistent with results of studies in other disease areas reporting that more convenient treatments tend to be associated with greater utility. ${ }^{15-17,19,21,22}$ During the interviews conducted for the current study, respondents often commented on the importance of treatment convenience in the context of the bone metastases health states, particularly when considering the impact of more intensive, time-consuming medical procedures.

The utility differences between SC injection and IV infusion were relatively small, ranging from roughly 0.01 to 0.03 , depending on the infusion duration and presence of chemotherapy. In comparison, health states representing different levels of symptom severity have repeatedly been found to have utility differences of at least $0.10 .{ }^{49-51}$ However, the current differences between injection and infusion may still be important because small differences in utility can have a substantial impact on the outcomes of a cost-utility 
Table 3 Results of $t$-tests comparing pairs of health states

\begin{tabular}{|c|c|c|c|c|c|}
\hline Comparison & Health states & Mean (SD) & Difference score & $t$-value (paired) & $P$-value \\
\hline \multicolumn{6}{|c|}{ Health states without chemotherapy } \\
\hline \multirow[t]{2}{*}{ B vs $C$} & B: Injection & $0.39(0.43)$ & 0.019 & 2.6 & 0.0106 \\
\hline & C: 30 -minute infusion & $0.38(0.44)$ & & & \\
\hline \multirow[t]{2}{*}{ B vs $D$} & B: Injection & $0.39(0.43)$ & 0.033 & 3.8 & 0.0002 \\
\hline & D: 2-hour infusion & $0.36(0.44)$ & & & \\
\hline \multirow[t]{2}{*}{ C vs D } & C: 30 -minute infusion & $0.38(0.44)$ & 0.013 & 3.1 & 0.0024 \\
\hline & D: 2-hour infusion & $0.36(0.44)$ & & & \\
\hline \multirow[t]{2}{*}{ C vs $E$} & C: 30 -minute infusion & $0.38(0.44)$ & 0.026 & 4.1 & $<0.0001$ \\
\hline & $\begin{array}{l}\text { E: } 30 \text {-minute infusion + renal monitoring } \\
\text { (blood draw on same day as infusion) }\end{array}$ & $0.35(0.43)$ & & & \\
\hline \multirow[t]{2}{*}{ C vs F } & C: 30 -minute infusion & $0.38(0.44)$ & 0.043 & 5.6 & $<0.0001$ \\
\hline & $\begin{array}{l}\text { F: } 30 \text {-minute infusion + renal monitoring } \\
\text { (blood draw } 2 \text { days prior to infusion) }\end{array}$ & $0.33(0.44)$ & & & \\
\hline \multirow[t]{2}{*}{ Evs $F$} & $\begin{array}{l}\text { E: } 30 \text {-minute infusion + renal monitoring } \\
\text { (blood draw on same day as infusion) }\end{array}$ & $0.35(0.43)$ & 0.017 & 2.9 & 0.0045 \\
\hline & $\begin{array}{l}\text { F: } 30 \text {-minute infusion }+ \text { renal monitoring } \\
\text { (blood draw } 2 \text { days prior to infusion) }\end{array}$ & $0.33(0.44)$ & & & \\
\hline \multicolumn{6}{|c|}{ Health states with chemotherapy } \\
\hline \multirow[t]{2}{*}{ G vs H } & G: Chemotherapy & $0.22(0.46)$ & 0.015 & 2.9 & 0.0041 \\
\hline & $\mathrm{H}:$ Injection + chemotherapy & $0.21(0.46)$ & & & \\
\hline \multirow[t]{2}{*}{ G vs I } & G: Chemotherapy & $0.22(0.46)$ & 0.027 & 3.7 & 0.0003 \\
\hline & I: 30-minute infusion + chemotherapy & $0.20(0.46)$ & & & \\
\hline \multirow[t]{2}{*}{ G vs J } & G: Chemotherapy & $0.22(0.46)$ & 0.036 & 4.4 & $<0.0001$ \\
\hline & J: 2-hour infusion + chemotherapy & $0.19(0.46)$ & & & \\
\hline \multirow[t]{2}{*}{ H vs I } & $\mathrm{H}$ : Injection + chemotherapy & $0.21(0.46)$ & 0.012 & 3.2 & 0.0019 \\
\hline & I: 30-minute infusion + chemotherapy & $0.20(0.46)$ & & & \\
\hline \multirow[t]{2}{*}{$\mathrm{H}$ vs J } & $\mathrm{H}$ : Injection + chemotherapy & $0.21(0.46)$ & 0.021 & 4.1 & $<0.0001$ \\
\hline & J: 2-hour infusion + chemotherapy & $0.19(0.46)$ & & & \\
\hline \multirow[t]{2}{*}{ I vs J } & I: 30 -minute infusion + chemotherapy & $0.20(0.46)$ & 0.009 & 3.1 & 0.0025 \\
\hline & J: 2-hour infusion + chemotherapy & $0.19(0.46)$ & & & \\
\hline
\end{tabular}

analysis when modeling a large number of patients over years of treatment.

The differences among the health state utilities followed logical patterns, indicating that participants understood the health states and the TTO task. Of all the treatment approaches represented in the health states, a monthly SC injection had the smallest impact on utility ( -0.004 without chemotherapy and -0.015 in the context of chemotherapy). The great majority $(\mathrm{n}=103[85.1 \%])$ of the 121 participants did not differentiate between health states A (no treatment modality) and B (with the injection) in the TTO task, indicating that the injection did not have a significant impact on health state preference. Among the 18 (14.9\%) who did differentiate between these two health states, $12(9.9 \%)$ preferred health state A because they preferred to avoid the inconvenience of the treatment. The other six respondents $(5.0 \%)$ preferred health state B because they thought the treatment would be of psychological benefit, even if there was no difference between the two health states in symptoms or health status.

Both the 30-minute and 2-hour IV infusions were associated with significantly greater disutility than SC injection (Table 3). Although the magnitude of the differences between the injection and infusion health state utilities tended to be small, they did reach statistical significance. Of the 121 respondents, 20 (16.5\%) differentiated between health states $\mathrm{B}$ (injection) and C (30-minute infusion), resulting in a utility difference of -0.019 between the two health states. Each additional inconvenience added to the infusion procedure led to an increased disutility. These inconveniences included a longer infusion process (health state D), a blood draw for renal monitoring occurring on the same day as the infusion (E), and a blood draw occurring 2 days prior to the infusion (F). Overall, this pattern of results suggests that small 
differences in treatment convenience can have an impact on preference and health state utility.

Chemotherapy was associated with a much greater disutility than any of the treatments focused on bone metastases. Of the 121 respondents, 88 (72.7\%) differentiated between the health states with and without chemotherapy (A vs G), resulting in a disutility of -0.17 for chemotherapy. Many of the respondents mentioned that their negative impression of chemotherapy was based not only on the health states themselves, but also on their observations of friends or family members who had received this type of treatment. This finding highlights strengths and weaknesses of the current study design. For example, reimbursement authorities often prefer that utilities are derived from general population samples, such as the sample in the current study, to ensure that societal values are represented when making decisions about public funding for medical treatment. ${ }^{41-43}$ However, it is not known whether a sample of patients with direct relevant experience would report different preferences than a general population sample. With regard to the current health states, it is possible that patients who had been treated with chemotherapy would be more or less accepting of its drawbacks than general population respondents who do not have direct experience with the treatment.

Like the use of the general population sample, vignettebased utility assessment methods also have strengths and limitations. In studies using this methodology, respondents rate health states based on brief vignettes, rather than direct personal experience. Although the vignettes for the current study were carefully drafted based on published literature and clinician experience with the treatment modality, the validity of each utility is limited by the level of detail and accuracy of the descriptions of the treatments. For example, the vignettes for the current study described each treatment as occurring once per month. However, the actual frequency of bisphosphonate infusions in real-world settings may vary across settings and patients, with some patients receiving treatment as often as every 3 weeks. ${ }^{5}$ Greater treatment frequency would likely be associated with greater inconvenience, which could influence a utility value. Unfortunately, the current vignettebased approach is unable to accurately capture this sort of variation among treatment experiences. Still, vignette-based utility assessment is often the best way to target the utilities of specific factors that may be difficult to isolate in a patient sample, such as the small differences in treatment modality, which were the focus of the current study. In contrast, generic instruments designed to derive utilities from patient samples, such as the EQ-5D ${ }^{45}$ or Health Utilities Index, ${ }^{52}$ may not have items or response options that are sensitive to such specific treatment attributes. ${ }^{53}$ The extent to which vignette-based utilities would correspond to utilities derived from direct patient experience is likely to vary depending on the medical condition and treatment in question.

The current findings show that treatment modality can have a significant effect on health state utility. The disutilities suggest that respondents perceived an inconvenience with each type of treatment for bone metastases, although SC injections were preferred over IV infusions, as indicated by the significant differences in mean utilities. The pattern of findings highlights the potential advantages of denosumab via $\mathrm{SC}$ injection in comparison with bisphosphonates, which are administered via intravenous infusions. The disutility associated with each treatment modality may be useful to include in cost-utility models estimating the value of treatments for patients with bone metastases.

\section{Acknowledgments}

The authors would like to thank Christine Thompson for statistical programming; Evan Davies, Andrew Palsgrove, Katie Devine, and Aria Gray for assistance with data collection; and Aria Gray and Fritz Hamme for production assistance.

\section{Disclosure}

Funding for this study was provided by Amgen, Inc, Thousand Oaks, CA, USA.

Three of the authors (Ze Cong, Karen Chung, and Ada Braun) are employees of Amgen and own stock in Amgen, but their input into the conceptualization and interpretation of this study represented their own opinions, rather than those of the company. Louis S Matza and Kelly McDaniel are employees of UBC, a company that received funding from Amgen for this research. At the time this study was conducted, Kate Van Brunt was also an employee of UBC. Alison Stopeck, Katia Tonkin, and Janet Brown did not receive funding for time spent contributing to this research. The authors report no other conflicts of interest in this work.

\section{References}

1. Coleman RE. Metastatic bone disease: Clinical features, pathophysiology and treatment strategies. Cancer Treat Rev. 2001;27(3): 165-176.

2. Smith HS. Painful osseous metastases. Pain Physician. 2011;14(4): E373-403.

3. Body JJ, Diel IJ, Bell R, et al. Oral ibandronate improves bone pain and preserves quality of life in patients with skeletal metastases due to breast cancer. Pain. 2004;111(3):306-312.

4. Weinfurt KP, Li Y, Castel LD, et al. The significance of skeletal-related events for the health-related quality of life of patients with metastatic prostate cancer. Ann Oncol. 2005;16(4):579-584. 
5. Aapro M, Abrahamsson PA, Body JJ, et al. Guidance on the use of bisphosphonates in solid tumours: recommendations of an international expert panel. Ann Oncol. 2008;19(3):420-432.

6. Coleman RE, Guise TA, Lipton A, et al. Advancing treatment for metastatic bone cancer: consensus recommendations from the Second Cambridge Conference. Clin Cancer Res. 2008;14(20):6387-6395.

7. Rosen LS, Gordon D, Tchekmedyian NS, et al. Long-term efficacy and safety of zoledronic acid in the treatment of skeletal metastases in patients with nonsmall cell lung carcinoma and other solid tumors: a randomized, Phase III, double-blind, placebo-controlled trial. Cancer. 2004;100(12):2613-2621.

8. ZOMETA ${ }^{\circledR}$ (zoledronic acid injection) [package insert]. East Hanover, NJ: Novartis Pharmaceuticals Corporation; 2012.

9. Pageau SC. Denosumab. MAbs. 2009;1(3):210-215.

10. Stopeck AT, Lipton A, Body JJ, et al. Denosumab compared with zoledronic acid for the treatment of bone metastases in patients with advanced breast cancer: a randomized, double-blind study. J Clin Oncol. 2010;28(35):5132-5139.

11. XGEVA ${ }^{\circledR}$ (denosumab injection) [package insert]. Thousand Oaks, CA: Amgen, Inc; 2013.

12. Fizazi K, Carducci M, Smith M, et al. Denosumab versus zoledronic acid for treatment of bone metastases in men with castrationresistant prostate cancer: a randomised, double-blind study. Lancet. 2011;377(9768):813-822.

13. Lipton A, Siena S, Rader M, et al. Comparison of denosumab versus zoledronic acid (ZA) for treatment of bone metastases in advanced cancer patients: An integrated analysis of 3 pivotal trials [Abstract]. Ann Oncol. 2010;21(Suppl 8):viii380.

14. Cavaliere CM, Chung KC. A cost-utility analysis of nonsurgical management, total wrist arthroplasty, and total wrist arthrodesis in rheumatoid arthritis. J Hand Surg Am. 2010;35(3):379-391.

15. Chancellor J, Aballéa S, Lawrence A, et al. Preferences of patients with diabetes mellitus for inhaled versus injectable insulin regimens. Pharmacoeconomics. 2008;26(3):217-234.

16. Huang ES, Shook M, Jin L, Chin MH, Meltzer DO. The impact of patient preferences on the cost-effectiveness of intensive glucose control in older patients with new-onset diabetes. Diabetes Care. 2006;29(2): 259-264.

17. Osborne RH, De Abreu Lourenço R, Dalton A, et al. Quality of life related to oral versus subcutaneous iron chelation: a time trade-off study. Value Health. 2007;10(6):451-456.

18. Polster M, Zanutto E, McDonald S, Conner C, Hammer M. A comparison of preferences for two GLP-1 products - liraglutide and exenatide - for the treatment of type 2 diabetes. J Med Econ. 2010;13(4):655-661.

19. Boye KS, Matza LS, Walter KN, Van Brunt K, Palsgrove AC, Tynan A. Utilities and disutilities for attributes of injectable treatments for type 2 diabetes. Eur J Health Econ. 2011;12(3):219-230.

20. Osborne RH, Dalton A, Hertel J, Schrover R, Smith DK. Healthrelated quality of life advantage of long-acting injectable antipsychotic treatment for schizophrenia: a time trade-off study. Health Qual Life Outcomes. 2012;10:35.

21. Teuffel O, Cheng S, Ethier MC, et al. Health-related quality of life anticipated with different management strategies for febrile neutropenia in adult cancer patients. Support Care Cancer. 2012;20(11): 2755-2764.

22. Feeny D, Townsend M, Furlong W, et al. Health-related quality-oflife assessment of prenatal diagnosis: chorionic villi sampling and amniocentesis. Genet Test. 2002;6(1):39-46.

23. Gage BF, Cardinalli AB, Albers GW, Owens DK. Cost-effectiveness of warfarin and aspirin for prophylaxis of stroke in patients with nonvalvular atrial fibrillation. JAMA. 1995;274(23):1839-1845.

24. Jewell EL, Smrtka M, Broadwater G, et al. Utility scores and treatment preferences for clinical early-stage cervical cancer. Value Health. 2011;14(4):582-586.

25. Wu JM, Fulton RG, Amundsen CL, Knight SK, Kuppermann M. Patient preferences for different severities of and treatments for overactive bladder. Female Pelvic Med Reconstr Surg. 2011;17(4):184-189.
26. Chapel HM, Spickett GP, Ericson D, et al. The comparison of the efficacy and safety of intravenous versus subcutaneous immunoglobulin replacement therapy. J Clin Immunol. 2000;20(2):94-100.

27. Chilton F, Collett RA. Treatment choices, preferences and decisionmaking by patients with rheumatoid arthritis. Musculoskeletal Care. 2008;6(1):1-14.

28. Robinson AM, McLean KA, Greaves M, Channer KS. Subcutaneous versus intravenous administration of heparin in the treatment of deep vein thrombosis; which do patients prefer? A randomized cross-over study. Postgrad Med J. 1993;69(808):115-116.

29. Matza LS, Van Brunt K, Chung KC, et al. Health state utilities for skeletal-related events associated with bone metastases. Paper presented at: ASCO 2011; June 3-7, 2011; Chicago, IL, USA.

30. Body JJ, Facon T, Coleman RE, et al. A study of the biological receptor activator of nuclear factor-kappaB ligand inhibitor, denosumab, in patients with multiple myeloma or bone metastases from breast cancer. Clin Cancer Res. 2006;12(4):1221-1228.

31. Lipton A, Zheng M, Seaman J. Zoledronic acid delays the onset of skeletal-related events and progression of skeletal disease in patients with advanced renal cell carcinoma. Cancer. 2003;98(5):962-969.

32. McKeage K, Plosker GL. Zoledronic acid: a pharmacoeconomic review of its use in the management of bone metastases. Pharmacoeconomics. 2008;26(3):251-268.

33. Saad F, Gleason DM, Murray R, et al; Zolendronic Acid Prostate Cancer Study Group. A randomized, placebo-controlled trial of zoledronic acid in patients with hormone-refractory metastatic prostate carcinoma. J Natl Cancer Inst. 2002;94(19):1458-1468.

34. Barrett-Lee P, Bloomfield D, Dougherty L, et al. An audit to determine the time taken to administer intravenous bisphosphonate infusions in patients diagnosed with metastatic breast cancer to bone in a hospital setting. Curr Med Res Opin. 2007;23(7):1575-1582.

35. Richhariya A, Qian Y, Zhao Y, Chung K. Time associated with intravenous zoledronic acid administration in patients with breast or prostate cancer and bone metastasis. Cancer Manag Res. 2012;4:55-60.

36. AREDIA $^{\circledR}$ (pamidronate disodium injection) [package insert]. East Hanover, NJ: Novartis Pharmaceuticals Corporation; 2011.

37. Conte P, Guarneri V. Safety of intravenous and oral bisphosphonates and compliance with dosing regimens. Oncologist. 2004;9 Suppl 4: $28-37$.

38. Abdulla A, Kapoor A. Emerging novel therapies in the treatment of castrate-resistant prostate cancer. Can Urol Assoc J. 2011;5(2): $120-133$.

39. Hassan MS, Ansari J, Spooner D, Hussain SA. Chemotherapy for breast cancer (Review). Oncol Rep. 2010;24(5):1121-1131.

40. Tannock IF, de Wit R, Berry WR, et al; TAX 327 Investigators. Docetaxel plus prednisone or mitoxantrone plus prednisone for advanced prostate cancer. N Engl J Med. 2004;351(15):1502-1512.

41. Brazier J. Valuing health states for use in cost-effectiveness analysis. Pharmacoeconomics. 2008;26(9):769-779.

42. NICE. Guide to the Methods of Technology Appraisal. London: National Institute for Health and Clinical Excellence; 2008. Available from: http://www.nice.org.uk/aboutnice/ howwework/devnicetech/GuideToMethodsTechnologyAppraisal2008. jsp?domedia=1\&mid=B52851A3-19B9-E0B5-D48284D172BD8459. Accessed May 10, 2013.

43. Pharmaceutical Benefits Advisory Committee (PBAC). Guidelines for Preparing Submissions to PBAC, Version 4.3. Canberra: Pharmaceutical Benefits Advisory Committee; 2008.

44. Brazier J, Ratcliffe J, Tsuchiya A, Salomon J. Measuring and Valuing Health Benefits for Economic Evaluation. New York, NY: Oxford University Press; 2007.

45. Dolan P, Gudex C, Kind P, Williams A. The time trade-off method: results from a general population study. Health Econ. 1996;5(2): 141-154.

46. Coleman RE. Clinical features of metastatic bone disease and risk of skeletal morbidity. Clin Cancer Res. 2006;12(20 Pt 2):6243s$6249 \mathrm{~s}$ 
47. Rowen D, Brazier J. Health utility measurement. In: Glied S, Smith PC, editors. The Oxford Handbook of Health Economics. New York, NY: Oxford University Press; 2011:788-813.

48. Matza LS, Boye KS, Yurgin N, et al. Utilities and disutilities for type 2 diabetes treatment-related attributes. Qual Life Res. 2007;16(7): 1251-1265.

49. Laupacis A, Bourne R, Rorabeck C, et al. The effect of elective total hip replacement on health-related quality of life. J Bone Joint Surg Am. 1993;75(11):1619-1626.

50. Chouinard G, Albright PS. Economic and health state utility determinations for schizophrenic patients treated with risperidone or haloperidol. J Clin Psychopharmacol. 1997;17(4):298-307.
51. Daly E, Gray A, Barlow D, McPherson K, Roche M, Vessey M. Measuring the impact of menopausal symptoms on quality of life. BMJ. 1993;307(6908):836-840.

52. Horsman J, Furlong W, Feeny D, Torrance G. The Health Utilities Index (HUI): concepts, measurement properties and applications. Health Qual Life Outcomes. 2003;1:54.

53. Garau M, Shah KK, Mason AR, Wang Q, Towse A, Drummond MF Using QALYs in cancer: a review of the methodological limitations. Pharmacoeconomics. 2011;29(8):673-685.

\section{Publish your work in this journal}

Patient Preference and Adherence is an international, peer-reviewed, open access journal focusing on the growing importance of patient preference and adherence throughout the therapeutic continuum. Patient satisfaction, acceptability, quality of life, compliance, persistence and their role in developing new therapeutic modalities and compounds to optimize clinical outcomes for existing disease states are major areas of interest. This journal has been accepted for indexing on PubMed Central. The manuscript management system is completely online and includes a very quick and fair peer-review system. Visit http://www.dovepress.com/ testimonials.php to read real quotes from published authors.

Submit your manuscript here: http://www.dovepress.com/patient-preference-and-adherence-journal 